\title{
A pro-inflammatory role for the Frizzled-8 receptor in chronic bronchitis
}

\author{
Anita I R Spanjer, ${ }^{1,2}$ Mark H Menzen, ${ }^{1,2}$ Akkelies E Dijkstra, ${ }^{2,3}$ Maarten van den \\ Berge, ${ }^{2,3}$ H Marike Boezen, ${ }^{2,4}$ David C Nickle, ${ }^{5}$ Don D Sin, ${ }^{6,7}$ Yohan Bossé, ${ }^{8,9}$ \\ Corry-Anke Brandsma, ${ }^{2,10}$ Wim Timens, ${ }^{2,10}$ Dirkje S Postma, ${ }^{2,3}$ Herman Meurs, ${ }^{1,2}$ \\ Irene H Heijink, ${ }^{2,3,10}$ Reinoud Gosens, ${ }^{1,2}$
}

\begin{abstract}
- Additional material is published online only. To view please visit the journal online (http://dx.doi.org/10.1136/ thoraxjnl-2015-206958)
\end{abstract}

For numbered affiliations see end of article.

\section{Correspondence to} Anita I R Spanjer, Department of Molecular Pharmacology, University of Groningen, Antonius Deusinglaan 1, Groningen 9713 AV, The Netherlands;

a.i.r.spanjer@rug.nl

Received 19 February 2015 Revised 24 November 2015 Accepted 21 December 2015 Published Online First 21 January 2016

\section{CrossMark}

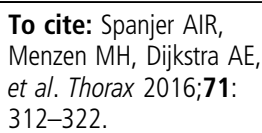

\section{ABSTRACT}

Rationale We have previously shown increased expression of the Frizzled-8 receptor of the Wingless/ integrase-1 (WNT) signalling pathway in COPD. Here, we investigated if the Frizzled-8 receptor has a functional role in airway inflammation associated with chronic bronchitis.

Methods Acute cigarette-smoke-induced airway inflammation was studied in wild-type and Frizzled-8deficient mice. Genetic association studies and lung expression quantitative trait loci (eQTL) analyses for Frizzled-8 were performed to evaluate polymorphisms in FZD8 and their relationship to tissue expression in chronic bronchitis. Primary human lung fibroblasts and primary human airway epithelial cells were used for in vitro studies

Results Cigarette-smoke-exposure induced airway inflammation in wild-type mice, which was prevented in Frizzled-8-deficient mice, suggesting a crucial role for Frizzled-8 in airway inflammation. Furthermore, we found a significant genetic association $(p=0.009)$ between single nucleotide polymorphism (SNP) rs663700 in the FZD8 region and chronic mucus hypersecretion, a characteristic of chronic bronchitis, in a large cohort of smoking individuals. We found SNP rs663700 to be a cis-eQTL regulating Frizzled-8 expression in lung tissue. Functional data link mesenchymal Frizzled-8 expression to inflammation as its expression in COPD-derived lung fibroblasts was regulated by pro-inflammatory cytokines in a genotype-dependent manner. Moreover, Frizzled-8 regulates inflammatory cytokine secretion from human lung fibroblasts, which in turn promoted MUC5AC expression by differentiated human airway epithelium.

Conclusions These findings indicate an important proinflammatory role for Frizzled-8 and suggest that its expression is related to chronic bronchitis. Furthermore, our findings indicate an unexpected role for fibroblasts in regulating airway inflammation in COPD.

\section{INTRODUCTION}

COPD is a complex lung disease, characterised by airflow limitation that is not fully reversible. Airflow limitation is usually progressive and associated with chronic inflammation, remodelling of the small airways and emphysema development in some patients. ${ }^{12}$

The heterogeneity of COPD is represented by different phenotypes within the patient population,

\section{Key messages}

What is the key question?

- What is the functional role for the Frizzled-8 receptor in airway inflammation in COPD?

\section{What is the bottom line?}

- We show that the Frizzled-8 receptor is associated with chronic bronchitis and is involved in cytokine secretion from human pulmonary fibroblasts as well as acute cigarette-smoke-induced inflammation in a mouse model.

\section{Why read on?}

- Our findings concerning the pro-inflammatory role for Frizzled-8 in airway inflammation in COPD provide a rationale for further exploration of the therapeutic potential of the Frizzled-8 receptor in COPD.

chronic bronchitis being one of them. Chronic bronchitis is defined by chronic cough and idiopathic sputum production for at least 3 months per year for two consecutive years. ${ }^{1}$ Inflammatory signals can cause mucus cell metaplasia, resulting in more mucus by both an increased production of mucus by goblet cells and decreased elimination from the airways. ${ }^{3}$ This is defined in patients as chronic mucus hypersecretion $(\mathrm{CMH})$. $\mathrm{CMH}$ is associated with bronchial inflammation, accelerated lung function decline, ${ }^{4}$ increased mortality ${ }^{5}$ and is a risk factor for COPD. ${ }^{6}$

COPD treatment is neither curative nor directed at different COPD phenotypes, including chronic bronchitis. At present, the pathophysiology and underlying mechanisms of chronic bronchitis are poorly understood. This hampers the development of targeted drugs. Therefore, a better understanding of the mechanisms behind chronic bronchitis is needed.

The Wingless/integrase-1 (WNT) signalling pathway plays an important role in lung development. ${ }^{7}$ Recent studies indicate involvement of the WNT signalling pathway in remodelling ${ }^{8} 9$ and inflammation ${ }^{10} 11$ in the lung. WNT ligands bind 
to transmembrane Frizzled (FZD) receptors, thereby controlling cell differentiation, growth and polarity. ${ }^{12}$ Our previous results showed that FZD8 expression is higher in pulmonary fibroblasts of patients with moderate (GOLD stage II) and severe (GOLD stage IV) COPD after stimulation with transforming growth factor (TGF)- $\beta$ compared with control fibroblasts. ${ }^{8}$ However, the functional role for FZD8 in COPD is as yet unknown. WNT-3A, ${ }^{10}$ WNT- $^{13}$ and WNT-5A ${ }^{14}$ have recently been shown to function as pro-inflammatory stimuli in the lung. Importantly, we previously showed that WNT-5A is a ligand for FZD8 in airway smooth muscle cells, ${ }^{9}$ reinforcing the possibility that the increased FZD8 expression in COPD fibroblasts may play a role in pro-inflammatory signalling in the lung.

Here, we aimed to investigate the link between FZD8 and airway inflammation in COPD. We studied the role of FZD8 in acute cigarette-smoke-induced airway inflammation in vivo using a mouse model and in vitro in human lung fibroblasts obtained from patients with COPD with and without CMH. Furthermore, we investigated the association between single nucleotide polymorphisms (SNPs) in the FZD8 region and $\mathrm{CMH}$, and their roles as cis-expression quantitative trait loci (eQTL), regulating FZD8 expression in lung tissue.

\section{METHODS}

A more extensive description of methods and statistics can be found in the online supplementary file.

\section{Ethics statement}

The study protocol followed national ethical and professional guidelines ('Code of conduct; Dutch federation of biomedical scientific societies'; http://www.federa.org) for all lung tissue and explant cell culture studies in Groningen. For the genetics study on $\mathrm{CMH}$, approval by the local medical ethics committee and written informed consent from all patients were obtained (see online supplementary file). All animal experiments were performed according to the national guidelines and approved by the University of Groningen Animal Ethical Committee (committee approval number 5912B).

\section{Animal studies}

Heterozygous, inbred, specified-pathogen-free breeding colonies FZD ${ }^{+/-}$mice $\left(\mathrm{C} 57 \mathrm{BL} / 6 ; 129 \mathrm{P} 2-F Z D 8^{\text {tm } 1 \text { Dgen }} / \mathrm{J}\right)$, showing no obvious phenotype, were obtained from The Jackson Laboratory (USA). After breeding, homozygous male FZD8 ${ }^{-/-}$

Table 1 Patient characteristics, patients with GOLD stage IV COPD

\begin{tabular}{lll}
\hline & $\begin{array}{l}\text { GOLD stage IV COPD } \\
\text { without CMH }\end{array}$ & $\begin{array}{l}\text { GOLD stage IV } \\
\text { COPD with CMH }\end{array}$ \\
\hline $\begin{array}{l}\text { Number of subjects } \\
\text { Age (range) }\end{array}$ & 8 & 8 \\
Sex & $58(57-62)$ & $54(48-61)$ \\
$\quad$ & \\
$\quad$ Male & 3 & 4 \\
$\quad$ Female & 5 & 4 \\
Pack-years (range) & $44.9(30-72)$ & $31.3(15-40)$ \\
Smoking status & Ex-smoker & Ex-smoker \\
\% predicted FEV1 (range) & $19.2(12.23-25.69)$ & $14.6(13.12-20.12)$ \\
FEV1/FVC (range) & $0.30(0.19-0.66)$ & $0.23(0.16-0.29)$ \\
Bronchiectasis & None & None \\
\hline Age, pack-years, predicted FEV1 and FEV1/FVC are represented as mean (range). \\
CMH, chronic mucus hypersecretion.
\end{tabular}

mice and wild-type ${ }^{+/+}$(WT) littermates were used for experiments as described in detail in the online supplementary file.

\section{Genetic association}

Genetic association studies for FZD8 and $\mathrm{CMH}$ were performed in the NELSON cohort; a large cohort of smoking individuals with and without COPD which was originally set up to detect lung cancer. ${ }^{15}$ After quality control, 717 heavy smokers with $\mathrm{CMH}$ (cases) and 1795 heavy smokers without $\mathrm{CMH}$ (controls) all with $\geq 20$ pack-years were included for the analysis as previously published. ${ }^{16}$ Six SNPs were tested using multivariate logistic regression analysis under an additive model, with adjustment for ex-smokers or current smokers and the two population sites (Groningen and Utrecht).

\section{eQTL analysis in lung tissue}

The lung eQTL analysis was performed in a large dataset of lung tissue samples where both genotype and genome-wide gene expression data are available through a collaboration of three universities (Laval University, Quebec City, Canada; University of British Columbia, Vancouver, Canada; University of Groningen, Groningen, the Netherlands), and Rosetta/Merck Sharpe and Dohme as has been described previously, including patient characteristics. ${ }^{17}$ After quality control, 1095 patients out of 1111 were included in the analysis. A cis-eQTL analysis was performed to determine the association between SNP rs663700 and the regulation of gene expression of genes within $100 \mathrm{~kb} .{ }^{17}$ In short, the association between genotype and expression of cis-eQTL genes was analysed using linear regression on imputed data, first in the three cohorts separately, followed by a meta-analysis. ${ }^{18} 19$

\section{Fibroblast and epithelial cell culture}

Human airway and parenchymal lung fibroblasts from ex-smoking patients with GOLD stage IV COPD with and without $\mathrm{CMH}$ were isolated from transplanted lungs as has been described previously. ${ }^{20}$ Presence of $\mathrm{CMH}$ was defined by patient records. Patient characteristics are shown in table 1 and showed no differences in age, gender, smoking status, lung function or medication use. At the same time, we determined that none of the patients used for the study in fibroblasts was diagnosed with bronchiectasis, excluding the possible influence on the data. MRC-5 human lung fibroblasts ${ }^{21}$ were obtained from Sigma (St Louis, Missouri, USA). Specific small interfering (si) RNA was used to knock down FZD8. Human airway epithelial cells were isolated from donor lungs. Cells were cultured and stimulated to measure gene expression using mRNA isolation and real-time PCR analysis, to perform immunohistochemistry for goblet cell number or to measure cytokine release using ELISA and Milliplex as described in detail in the online supplementary file.

\section{Statistical analysis}

Statistical analysis of in vitro and in vivo experiments was performed with the SigmaPlot software (Systat Software, San Jose, California, USA). All real-time PCR data were log-transformed before analysis. For comparison between two conditions, the Student's t test $(\alpha=0.05$, tested two-tailed $)$ was used. Differences in FZD8 gene expression in fibroblasts between two genotypes were tested using a one-tailed Mann-Whitney test, based on the results of the eQTL analysis. To test differences between different patient groups, one-way analysis of variance (ANOVA) on ranks was used. For comparison between multiple conditions within the primary MRC-5 human lung fibroblast 
experiments and within the different groups of mice, two-way ANOVA was used. Tests used for the analyses of the genetic studies are described in the appropriate sections. Where appropriate, ANOVA was followed by a post hoc analysis using the Student-Newman-Keuls multiple comparisons test. $\mathrm{p}<0.05$ was considered significant.

\section{RESULTS \\ FZD8 regulates cigarette-smoke-induced airway inflammation in mice}

Inflammation in COPD follows a characteristic pattern of increased numbers of macrophages, lymphocytes and neutrophils. $^{2}$ To investigate a possible role for FZD8 in inflammation, we used $\mathrm{FZD}^{-1-}$ mice and studied acute cigarette-smoke-induced airway inflammation, as described previously. ${ }^{22}$ FZD8 $^{-/}$mice were born according to normal Mendelian ratio and survived after birth with no differences compared with WT mice. Baseline lung structure was not different for the FZD8 ${ }^{-/}$mice and WT mice in terms of gross morphological structure and expression of $\alpha$-sm-actin around the airways and vessels (see online supplementary figures S1A-C). Baseline presence of inflammatory cells and pro-inflammatory cytokines was slightly higher in $\mathrm{FZD}^{-/}$mice compared with WT mice (figure $1 \mathrm{~A}-\mathrm{G}$ ) and this was significant for neutrophil number $(p=0.004)$ and CXCL5 $(p=0.005)$ gene expression when using a Student's $t$ test on the air-exposed animals only.

Four days of cigarette-smoke exposure induced an increase in the number of lymphocytes and neutrophils in the bronchial alveolar lavage fluid (BALF) of WT mice (figure 1A, B). No effect of cigarette smoke was found on total cells and macrophages (figure 1C, D). Notably, cigarette smoke did not increase the number of lymphocytes and neutrophils in $\mathrm{FZD}^{-/-}$mice and neutrophil number in cigarette-smoke-exposed $\mathrm{FZD} 8^{-/-}$mice was significantly lower compared with cigarette-smoke-exposed WT mice (figure 1B). Keratinocyte-derived cytokine (KC), CXCL2 and CXCL5 are chemotactic for neutrophils, and the gene expression of all three cytokines was increased upon cigarette-smoke exposure in WT mice (figure 1E-G). KC and CXCL2 tended to be reduced in $\mathrm{FZD}^{-/-}$mice, whereas for CXCL5, the cigarette-smoke-induced increase was completely absent in FZD $8^{-/-}$mice. Gene expression of FZD8 and its putative ligands WNT-5A and WNT-5B was also measured in whole lung homogenates and was not different between groups, although small, nonsignificant increases in cigarette-smoke-exposed WT mice were apparent for all three genes (see online supplementary figures S1D-F). MUC5AC gene expression significantly increased after cigarette-smoke exposure in WT mice but not in $\mathrm{FZD}^{-/}$mice (see online supplementary figure S1G). However, consistent with previous studies, 22 we did not find any cells positive for MUC5AC protein expression in lung tissue sections before and after cigarette-smoke exposure in WT and $\mathrm{FZD}^{-/-}$mice. Together, these results suggest that FZD8 is involved in acute cigarette-smoke-induced airway inflammation in mice in vivo.

\section{A genetic association between FZD8 and CMH}

In view of this pro-inflammatory role, we next studied the role of FZD8 in chronic bronchitis and found a genetic association between SNP rs663700 in the FZD8 region and CMH $(p=0.009 ; O R=0.8059 ; 0.684-0.9495)$ in a large cohort of smoking individuals with and without COPD, implying that FZD8 may play a role in the development of $\mathrm{CMH}$ (figure 2A). Notably, we found that SNP rs663700 was associated with $\mathrm{CMH}$ already in the healthy smokers of the cohort $(n=1348$; $\mathrm{p}=0.002$ ), indicating that the SNP links to CMH specifically and is independent of the presence of COPD. The protective $\mathrm{C}$ allele is associated with lower risk of having $\mathrm{CMH}$ and the susceptibility allele $\mathrm{T}$ with higher risk of having $\mathrm{CMH}$. The five other SNPs tested in the FZD8 region were less or not associated with $\mathrm{CMH}$ (see online supplementary table S3).

Next, we performed an eQTL analysis in a large dataset of lung tissue samples of non-smokers, current smokers and ex-smokers with and without COPD ${ }^{17}$ to determine the association between SNP rs663700 and the lung mRNA expression levels of genes within $100 \mathrm{~kb}$, as has been described previously. ${ }^{17}$ The eQTL analysis showed that SNP rs663700 has a cis-eQTL effect on FZD8 $\left(\mathrm{p}=4.58 \times 10^{-11}\right)$ and CCNY (Cyclin $\left.Y ; p=4.72 \times 10^{-4}\right)$ gene expression in lung tissue $(n=1095)$. The strongest eQTL effect was on FZD8 where the protective C allele is associated with lower expression and the susceptibility allele $\mathrm{T}$ with higher expression of FZD8 (results from the meta-analysis across the three cohorts: $p=4.94 \times 10^{-10}$; Spearman $\rho=0.19$; figure $2 \mathrm{~B}-\mathrm{E}$ ). The allele frequency within the study population was $65.8 \%$ for the homozygous CC, $30.3 \%$ for the heterozygous CT and $3.9 \%$ for the homozygous TT.

\section{FZD8 expression in lung fibroblasts is associated with $\mathrm{CMH}$}

We previously established that FZD8 is expressed in fibroblasts and that its expression is increased in COPD. ${ }^{8}$ To link these previous findings with the current observation that FZD8 regulates inflammation and is involved in chronic bronchitis, we studied the pro-inflammatory role of FZD8 in human airway and parenchymal lung fibroblasts from ex-smoking patients with GOLD stage IV COPD with and without CMH. We found that interleukin (IL)-1 $\beta$ and epidermal growth factor (EGF) induced a strong increase in FZD8 gene expression in lung fibroblasts. Interestingly, this normalised increase in FZD8 gene expression was stronger in primary airway and parenchymal lung fibroblasts of patients with GOLD stage IV COPD with $\mathrm{CMH}$ than in those without $\mathrm{CMH}$ (figure 3A, C). The mean basal FZD8 gene expression did not differ with respect to $\mathrm{CMH}$ status. No differences in gene expression with respect to CHM status were observed for the effects of IL-1 $\beta$ or EGF on WNT-5A, WNT-5B, WNT-16, FZD2 and FZD6 gene expression in parenchymal lung fibroblasts (see online supplementary figures S2A, B), indicating a specific effect on FZD8. Although TGF- $\beta$ did augment FZD8 gene expression, there was no significant difference between the two patient groups regarding $\mathrm{CMH}$ status, whereas tumour necrosis factor (TNF)- $\alpha$ and cigarette-smoke extract did not augment FZD8 gene expression (see online supplementary figure S2C). This indicates a specific effect for IL-1 $\beta$ and EGF. Interestingly, receptor expression of EGFR was higher in airway fibroblasts and pulmonary fibroblasts of patients with GOLD stage IV COPD with $\mathrm{CMH}$ than without $\mathrm{CMH}$, as well as IL1R1 expression in airway fibroblasts of patients with GOLD stage IV COPD with $\mathrm{CMH}$ than without $\mathrm{CMH}$ (figure $3 \mathrm{~B}, \mathrm{D}$ ), suggesting a link between receptor expression and functional FZD8 induction by IL-1 $\beta$ and EGF.

\section{FZD8 gene expression in lung fibroblasts is regulated by SNP rs663700}

We further analysed the genetic basis of FZD8 gene expression in airway and parenchymal lung fibroblasts of patients with GOLD stage IV COPD with and without CMH. The fold induction of FZD8 gene expression after stimulation with IL-1 $\beta$ and EGF was found to be dependent on the genotype of the patients with respect to SNP rs663700. In line with the results of our lung eQTL analysis, airway fibroblasts of patients with GOLD 

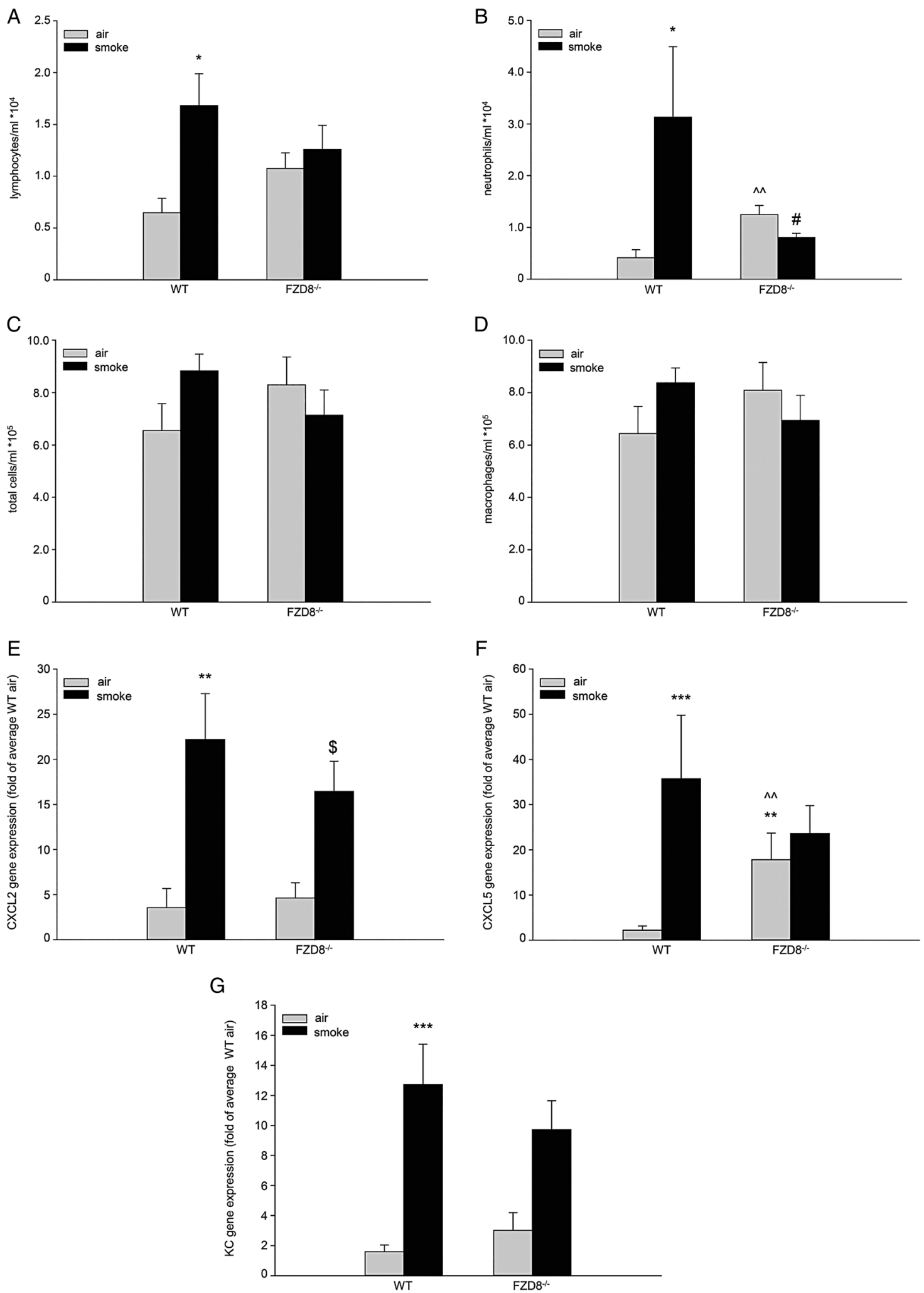

Figure 1 Acute cigarette-smoke-induced airway inflammation is dependent on FZD8. Wild-type (WT) and FZD8 ${ }^{-l-}$ mice were exposed to cigarette smoke for 4 days. (A) Lymphocyte numbers in bronchial alveolar lavage fluid (BALF); $p=0.068$ for the interaction of smoke exposure and genotype (two-way ANOVA). (B) Neutrophil numbers in BALF; $p=0.035$ for the interaction of smoke exposure and genotype (two-way ANOVA). (C) Total cell number in BALF; $p=0.079$ for the interaction of smoke exposure and genotype (two-way ANOVA). (D) Macrophage numbers in BALF; $p=0.105$ for the interaction of smoke exposure and genotype (two-way ANOVA). (E) CXCL2 gene expression in whole lung homogenates; $p=0.343$ for the interaction of smoke exposure and genotype (two-way ANOVA). (F) CXCL5 gene expression in whole lung homogenates; $p=0.021$ for the interaction of smoke exposure and genotype (two-way ANOVA). (G) KC gene expression in whole lung homogenates. Data represent mean $\pm S E M$ of eight mice per group. * $p<0.05$, ${ }^{* *} p<0.01,{ }^{* * *} p<0.001$ compared with air-exposed WT mice ${ }^{\#} p<0.05$ compared with smoke-exposed WT mice ${ }^{\$} p<0.05$ compared with air-exposed $\mathrm{FZD}^{-1-}$ mice (two-way ANOVA with Student-Newman-Keuls multiple comparisons test). $\wedge \wedge \mathrm{p}<0.01$ compared with air-exposed FZD $8^{-1-}$ mice (two-tailed Student's $t$ test). ANOVA, analysis of variance; $C X C L$, chemokine ligand; $K C$, keratinocyte-derived cytokine; EGF, epidermal growth factor. 

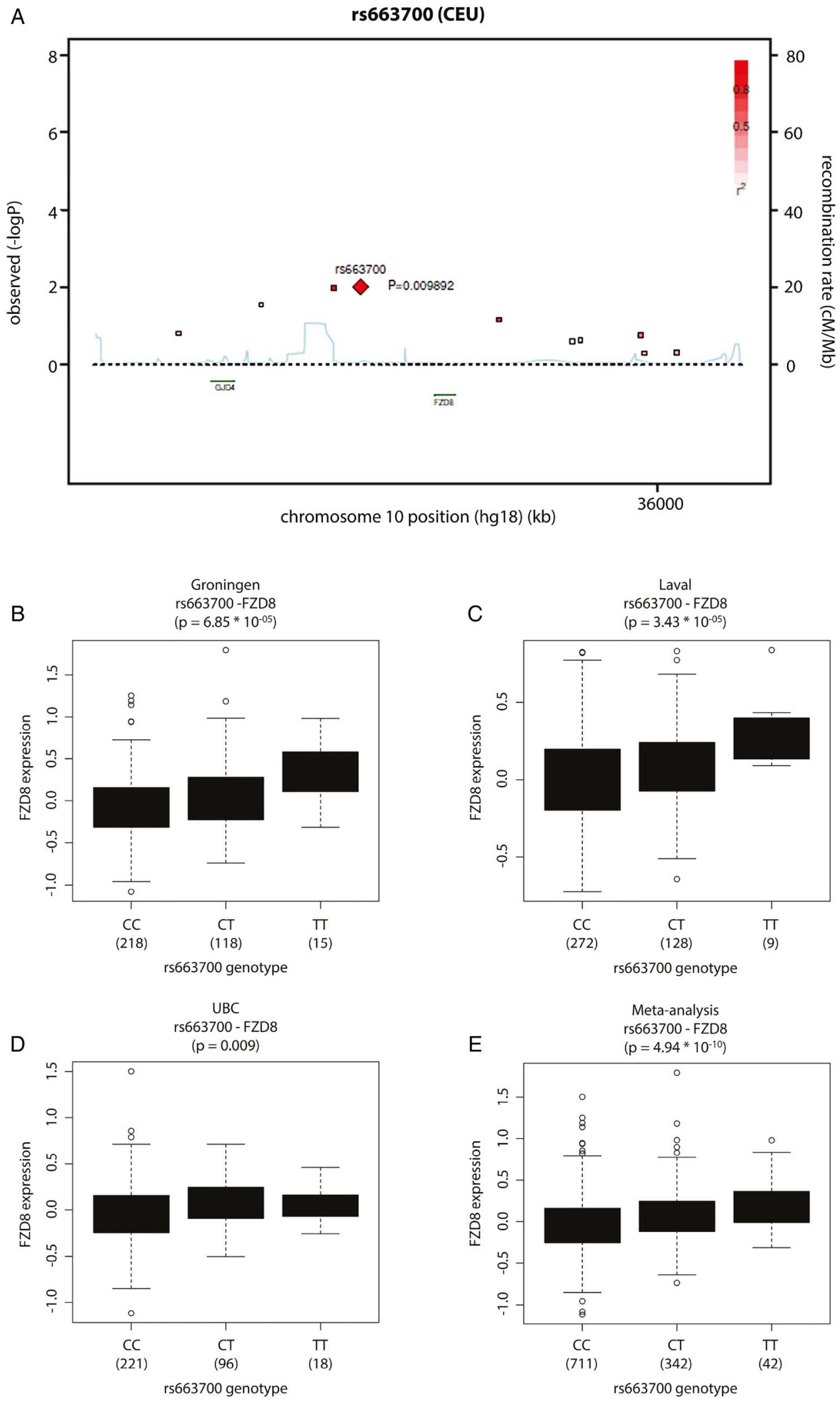

Figure 2 Genetic association between FZD8 and chronic mucus hypersecretion (CMH). (A) Genetic association between single nucleotide polymorphism (SNP) rs663700 in the FZD8 region and CMH within a cohort of heavy smokers with and without COPD ( $\mathrm{n}=717$ with CMH and $\mathrm{n}=1795$ without $\mathrm{CMH}$ ); $\mathrm{p}=0.009 ; \mathrm{OR}=0.8059 ; 0.684-0.9495$. FZD8 and SNP rs663700 are located on chromosome 10. C is the wild-type (WT) allele and protective, and T is the variant, susceptibility allele of SNP rs663700. CEU stands for Caucasian population in the HapMap project. (B) Expression quantitative trait loci (eQTL) analysis results from the Groningen cohort $(n=363)$. Boxplots show the relationship between gene expression and genotype; $p=6.85 \times 10^{-05}$. (C) eQTL analysis results from the Laval cohort $(n=409)$. Boxplots show the relationship between gene expression and genotype; $p=3.43 \times 10^{-05}$. (D) eQTL analysis results from the UBC cohort $(n=339)$. Boxplot show the relationship between gene expression and genotype UBC; $p=0.009$. (E) eQTL analysis results from the meta-analysis across the three cohorts. Boxplots show the relationship between gene expression and genotype; $p=4.94 \times 10^{-10}$. 

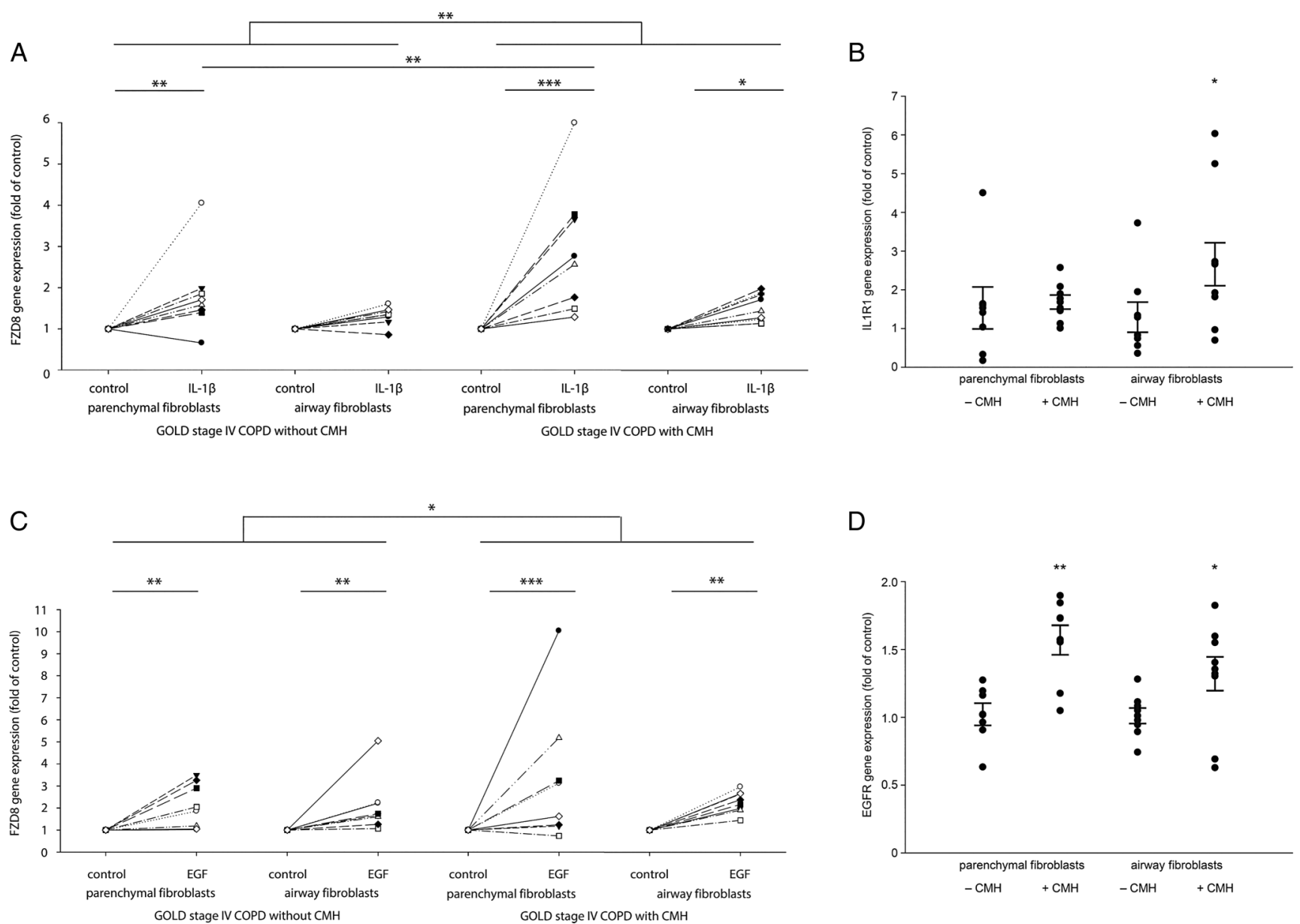

Figure 3 Interleukin (IL)-1 $\beta$ - and epidermal growth factor (EGF)-induced FZD8 expression is associated with chronic mucus hypersecretion (CMH) Primary airway and parenchymal lung fibroblasts of patients with GOLD stage IV COPD with and without CMH were stimulated for $4 \mathrm{~h}$ with IL-1 $\beta$ $(1 \mathrm{ng} / \mathrm{mL})$ or EGF $(10 \mathrm{ng} / \mathrm{mL})$ to study FZD8 gene expression. Basal delta Cq-values did not differ between groups: parenchymal fibroblasts GOLD stage IV COPD: $16.29 \pm 0.61$, airway fibroblasts GOLD stage IV COPD: $16.28 \pm 0.28$, parenchymal fibroblasts GOLD stage IV COPD with CMH: 15.96 \pm 0.38 , airway fibroblasts GOLD stage IV COPD with CMH: $15.99 \pm 0.51$. (A) IL-1 $\beta$-induced FZD8 gene expression. (B) Basal IL1R1 gene expression. (C) EGF-induced FZD8 gene expression. (D) Basal EGFR gene expression. Data represent induction of gene expression of eight patients per group. ${ }^{*} p<0.05,{ }^{* *} p<0.01$ (one-way ANOVA with Student-Newman-Keuls multiple comparisons test). ANOVA, analysis of variance.

stage IV COPD with the CT variant showed a higher induction of FZD8 gene expression than did patients with the CC variant after IL-1 $\beta$ but not after EGF stimulation (figure 4A, B). In parenchymal lung fibroblasts, this effect was less pronounced (see online supplementary figure S3A, B). The low allele frequency (3.9\%) of the TT variant precluded us from obtaining sufficient numbers of donors to analyse the impact of the TT variant on gene expression in these studies. Our results suggest that variants in FZD8 gene expression in lung fibroblasts play a role in patients with COPD who have $\mathrm{CMH}$.

\section{FZD8 regulates IL-6 and CXCL8 secretion by lung fibroblasts}

In view of our findings using the FZD $8^{-1-}$ mice, our next aim was to investigate in what way the changed expression in FZD8 gene expression in lung fibroblasts may affect pro-inflammatory responses. We investigated which cytokines are produced by primary MRC-5 human lung fibroblasts in response to IL-1 $\beta$ and EGF. Stimulation with IL-1 $\beta$-induced granulocyte colony-stimulating factor (G-CSF), granulocyte macrophage CSF (GM-CSF), IL-6, chemokine ligand (CXCL)8, interferon gamma-induced protein (IP)-10 and monocyte chemotactic protein (MCP)-1 secretion (figure 5A). Stimulation with EGF induced GM-CSF, IL-6 and CXCL8 secretion (figure 5B). We hypothesised that FZD8 may regulate the IL-1 $\beta$-induced and EGF-induced release of IL-6 and CXCL8 from fibroblasts, as these cytokines are increased in COPD and contribute largely to the inflammation seen in COPD. ${ }^{24}$ Moreover, in accordance with a previous study, ${ }^{25}$ we observed that IL-6 and CXCL8 induce MUC5AC gene expression and CXCL8 induces PAS positive mucin protein expression indicative of goblet cell differentiation in differentiated primary human airway epithelial cells from healthy donors (figures 5C, D), further linking these cytokines to $\mathrm{CMH}$. We were, however, not able to detect MUC5AC protein release. We found that IL- $1 \beta$ dose dependently increased both IL-6 and CXCL8 secretion from primary MRC-5 human lung fibroblasts (figure 6A, B), whereas EGF dose dependently increased CXCL8 secretion (figure 6C), but not IL-6 secretion (see online supplementary figure S4A). FZD8 knock-down by specific siRNA (knockdown efficiency $65 \%$ on average; see online supplementary figures $\mathrm{S} 4 \mathrm{~B}, \mathrm{C})$ significantly reduced IL-1 $\beta$-induced IL- 6 secretion $(p=0.037$ for the interaction of IL-1 $\beta$ stimulation and siRNA knock-down (two-way ANOVA) and CXCL8 secretion ( $p=0.008$ for the interaction of IL-1 $\beta$ stimulation and siRNA knock-down (two-way ANOVA)) (figures 6A, B). EGF-induced CXCL8 secretion was also reduced by FZD8 knock-down $(p<0.001$ for the interaction of 

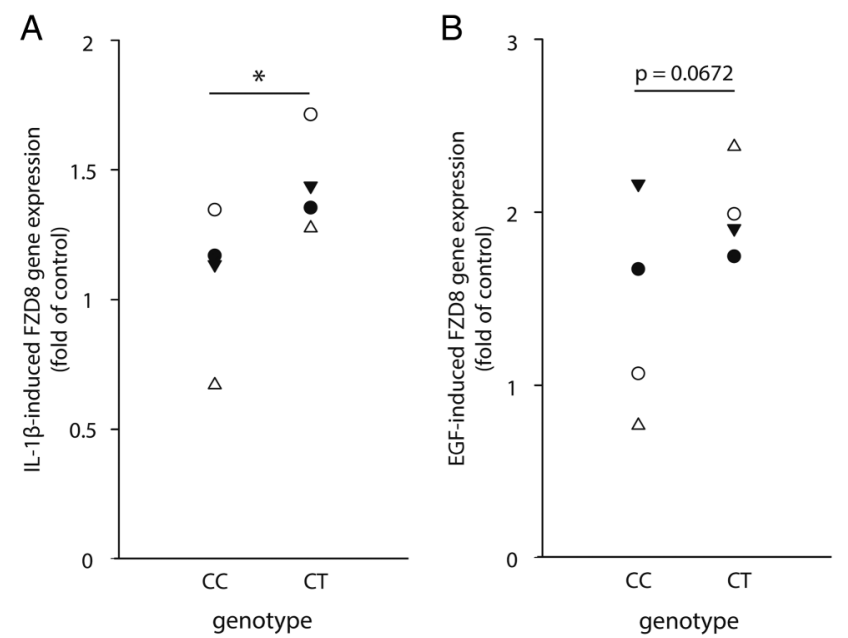

Figure 4 Interleukin (IL)-1 $\beta$ - and epidermal growth factor (EGF)-induced increase of FZD8 gene expression in patients with GOLD stage IV COPD with and without chronic mucus hypersecretion (CMH) is regulated by single nucleotide polymorphism (SNP) rs663700. Relationship between the fold induction in FZD8 gene expression in airway fibroblasts of patients with GOLD stage IV COPD after stimulation with IL-1 $\beta$ or EGF and the FZD8 genotype of the patients, with regard to SNP rs663700. (A) IL-1 $\beta$-induced FZD8 gene expression. (B) EGF-induced FZD8 gene expression. The experiment was performed for eight patients per group, four of which had a known genotype. Data represent four patients with CC and four patients with CT. ${ }^{*} p<0.05$ (one-tailed Mann-Whitney test).

EGF stimulation and siRNA knockdown (two-way ANOVA)) (figure 6C). These data show that FZD8 is involved in the IL-1 $\beta$-induced secretion of IL- 6 and CXCL8 as well as in the EGF-induced secretion of CXCL8. Furthermore, these data stress the importance of the fibroblast in the inflammatory response as seen in chronic bronchitis in COPD.

\section{DISCUSSION}

This study shows for the first time that FZD8 plays a critical role in inflammatory processes involved in chronic bronchitis, including $\mathrm{CMH}$, and reveals an unexpected role for the fibroblast in this process. We found that acute cigarette-smoke-induced airway inflammation in an in vivo mouse model is partly FZD8 dependent. In FZD $8^{-1-}$ mice, cigarette smoke did not induce neutrophilic inflammation, which was accompanied by the reduced expression of CXCL5 and to a lesser extent of CXCL2 and KC compared with cigarette-smoke-exposed WT mice. Furthermore, we show that there is an association between pro-inflammatory cytokine-induced FZD8 gene expression in airway and parenchymal lung fibroblasts of patients with GOLD stage IV COPD and the presence of $\mathrm{CMH}$. This is explained in part by increased EGFR and IL1R1 receptor expression in fibroblasts of patients with GOLD stage IV COPD with CMH compared with patients without $\mathrm{CMH}$ and in part by a polymorphism in the FZD8 region. SNP rs663700 in the FZD8 region is associated with $\mathrm{CMH}$ in a cohort of smokers and additionally is a cis-eQTL in lung tissue, regulating FZD8 expression. Moreover, SNP rs663700 is associated with increased FZD8 gene expression in fibroblasts of patients with GOLD stage IV COPD with $\mathrm{CMH}$. Upon stimulation with IL-1 $\beta$ and EGF, primary MRC-5 human lung fibroblasts secrete IL-6 and CXCL8, a process which is regulated by FZD8, and we show that both of these cytokines are able to initiate MUC5AC gene expression and CXCL8 induces goblet cells in differentiated primary human airway epithelial cells.

COPD is a heterogeneous disease characterised by different patient subpopulations. ${ }^{26}$ We investigated the potential role for FZD8 in chronic bronchitis, and found a clear association between the cytokine-induced expression of FZD8 gene expression and $\mathrm{CMH}$, a clinical expression which is a consequence of the pathologic process in chronic bronchitis. Of importance is to emphasise that the genetic association was independent of the presence of COPD, indicating that FZD8 likely plays a role in $\mathrm{CMH}$ in healthy smokers as well. $\mathrm{CMH}$ is associated with bronchial inflammation, accelerated lung function decline, COPD morbidity and increased mortality. ${ }^{4-6}$ The association of the genetic variance in FZD 8 with $\mathrm{CMH}$ is the first evidence to support a genetic link between the WNT signalling pathway and a phenotype of COPD. Interestingly, we also found a specific role for FZD8 in the chronic bronchitis phenotype, reinforcing the contention that phenotypes of COPD may be characterised by specific pathophysiological mechanisms.

While the communication of both damaged and intact epithelium to the fibroblasts is widely recognised in the airways, ${ }^{27}$ we now find indications that fibroblasts play an additional crucial role in inflammation and epithelial mucus production. Upon damage, the epithelium secretes inflammatory cytokines such as EGF, IL-1 $\beta$ and IL- $1 \alpha$. IL- $1 \alpha$ has been shown to induce IL- 6 and CXCL8 in fibroblasts. ${ }^{28}$ Importantly, IL-6 and CXCL8 secretion from primary MRC-5 human lung fibroblasts are increased upon coculturing these cells with human bronchial epithelial cells. This process is mediated via IL- $1 \alpha .{ }^{29}$ IL- $1 \alpha$ and IL- $1 \beta$ are known to function via the same receptor, IL1R1. IL-1 $\beta$ and EGF stimulated fibroblasts to produce IL-6 and CXCL8 via FZD8 in our study. We propose that in this way, FZD8 contributes to the inflammation present in chronic bronchitis. Our data support this hypothesis, as neutrophil and lymphocyte recruitment in the BALF are reduced in $\mathrm{FZD}^{-/-}$mice as well as mRNA expression of the neutrophil attractants CXCL5 and KC in whole lung homogenates, whereas IL-6-induced and CXCL8-induced mucus production in differentiated primary human airway epithelial cells. Both cytokines are secreted by human lung fibroblasts upon stimulation with IL-1 $\beta$ and EGF, and we demonstrate that this process is regulated by FZD8. In addition, we show that expression of IL1R1 and the EGFR is increased in fibroblasts of patients with GOLD stage IV COPD with $\mathrm{CMH}$ compared with patients without $\mathrm{CMH}$. This shows that IL-1 $\beta$ and EGF lead to increased FZD8 expression in CMH in part via increased expression of their receptors, and in part based on FZD8 genotype.

For many WNT ligands, it is either not known or highly context dependent via which FZD receptor they exert their effect. FZD8 is a known receptor for WNT-5 $\mathrm{A}^{9}$ and WNT-5B $\mathrm{B}^{30}$ in airway smooth muscle cells and pulmonary fibroblasts. However, based on the crystal structure of the cysteine-rich domain of FZD8, it must be assumed that most if not all WNT ligands are potential agonists, ${ }^{31}$ whereas $\mathrm{R}$-spondins have also been described as FZD8 agonists. ${ }^{32}$ Conversely, connective tissue growth factor ${ }^{33}$ and insulin-like growth-factor-binding protein- $4^{34}$ may antagonise FZD8 by direct binding. WNT-5A and WNT-5B are highly expressed in fibroblasts, ${ }^{8}$ and a role of FZD8 in their responses has been described. ${ }^{9}{ }^{30}$ Furthermore, the regulation by WNT-5A of CXCL8 release from human neutrophils has been described. ${ }^{14}$ Therefore, WNT-5A and WNT-5B are potential ligands for FZD8 in chronic bronchitis, which is supported by our findings that WNT-5A and WNT-5B 

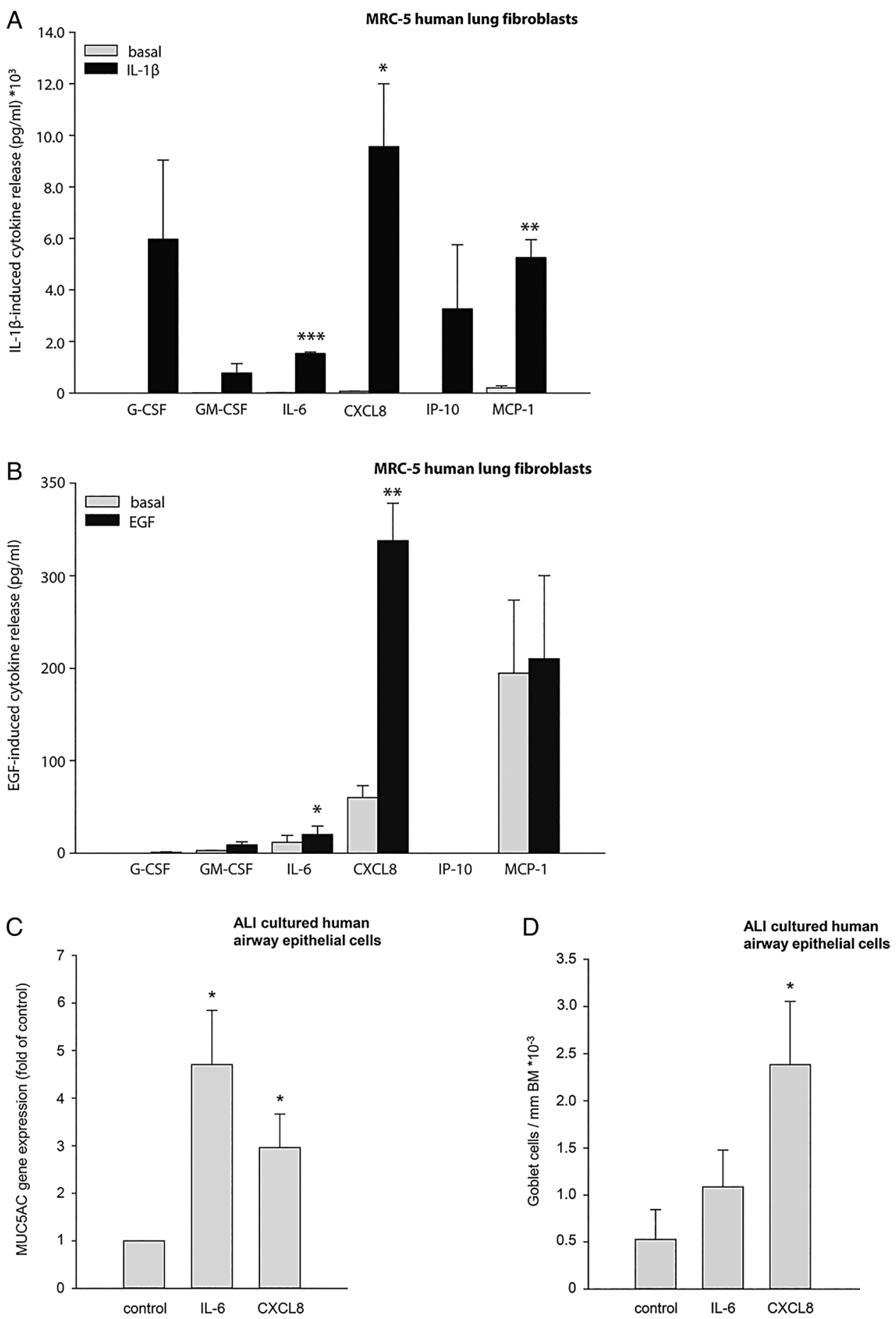

Figure 5 Interleukin (IL)- 6 and CXCL8 are released by primary MRC-5 human lung fibroblasts and induce MUC5AC gene expression in ALI-cultured primary human airway epithelial cells. Primary MRC-5 human lung fibroblasts were stimulated for $24 \mathrm{~h}$ with IL-1 $\beta$ (1 ng/mL) or epidermal growth factor (EGF) $(10 \mathrm{ng} / \mathrm{mL})$. A Milliplex was performed to screen for 26 cytokines to investigate which cytokines were produced. (A) IL-1 $\beta$-induced cytokine secretion from primary MRC-5 human lung fibroblasts. (B) EGF-induced cytokine secretion from primary MRC-5 human lung fibroblasts. Data represent mean \pm SEM of five independent experiments. ${ }^{*} p<0.05,{ }^{* *} p<0.01,{ }^{* *} p<0.001$ compared with basal conditions (two-tailed Student's $\mathrm{t}$ test). Primary human airway epithelial cells were stimulated for $24 \mathrm{~h}$ with IL-6 $(10 \mathrm{ng} / \mathrm{mL})$ and CXCL8 $(10 \mathrm{ng} / \mathrm{mL})$ to study MUC5AC gene expression. (C) IL-6- and CXCL8-induced MUC5AC gene expression in differentiated primary human airway epithelial cells. Data represent mean \pm SEM of five independent experiments. (D) IL-6- and CXCL8-induced number of goblet cells per mm basement membrane (BM) in paraffin-embedded sections of differentiated primary human airway epithelial cells. Data represent mean \pm SEM of four independent experiments. ${ }^{*} \mathrm{p}<0.05$ compared with basal conditions (two-tailed Student's $t$ test).

induce IL-6 and CXCL-8 release from human lung fibroblasts. ${ }^{35}$ Clearly, a better appreciation of the ligands involved in FZD8 signalling, their cellular sources and their role in chronic bronchitis require further studies.
COPD is currently treated symptomatically and not phenotype specific. The awareness that patients with COPD need treatment according to their phenotype stimulated the investigation of new drug targets. ${ }^{24}$ We found IL- 6 and CXCL8 to be important in 
Figure 6 FZD8 regulates interleukin (IL)-1 $\beta$ - and epidermal growth factor (EGF)-induced IL-6 and CXCL8 secretion. Primary MRC-5 human lung fibroblasts were transfected with specific FZD8 siRNA or non-targeting siRNA and stimulated for $24 \mathrm{~h}$ with increasing concentrations of IL-1 $\beta$ (0.03-3 $\mathrm{ng} / \mathrm{mL})$ and EGF $(0.1-10 \mathrm{ng} /$ $\mathrm{mL}$ ). (A) IL-1 $\beta$-induced IL-6 secretion by primary MRC -5 human lung fibroblasts; $p=0.037$ for the interaction of IL-1 $\beta$ stimulation and siRNA knock-down (two-way analysis of variance (ANOVA)). (B) IL-1 $\beta$-induced CXCL8 secretion by primary MRC- 5 human lung fibroblasts; $p=0.008$ for the interaction of IL-1 $\beta$ stimulation and siRNA knock-down (two-way ANOVA). (C) EGF-induced CXCL8 secretion by primary MRC-5 human lung fibroblasts; $p<0.001$ for the interaction of EGF stimulation and siRNA knock-down (two-way ANOVA). Data represent mean \pm SEM of five (CXCL8) or four (IL-6) independent experiments. ${ }^{* *} p<0.01,{ }^{* * *} p<0.001$ compared with control ${ }^{\#} p<0.05$ compared with stimulated control siRNA (two-way ANOVA with Student-Newman-Keuls multiple comparisons test).

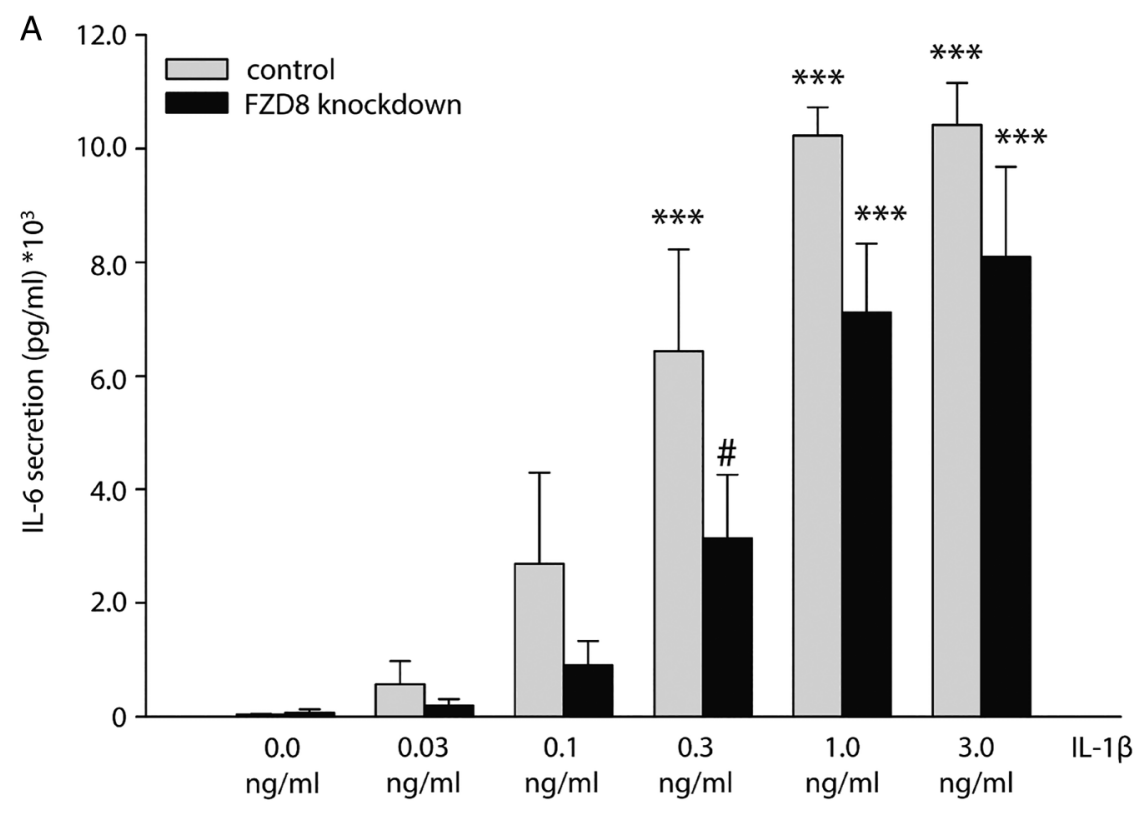

B
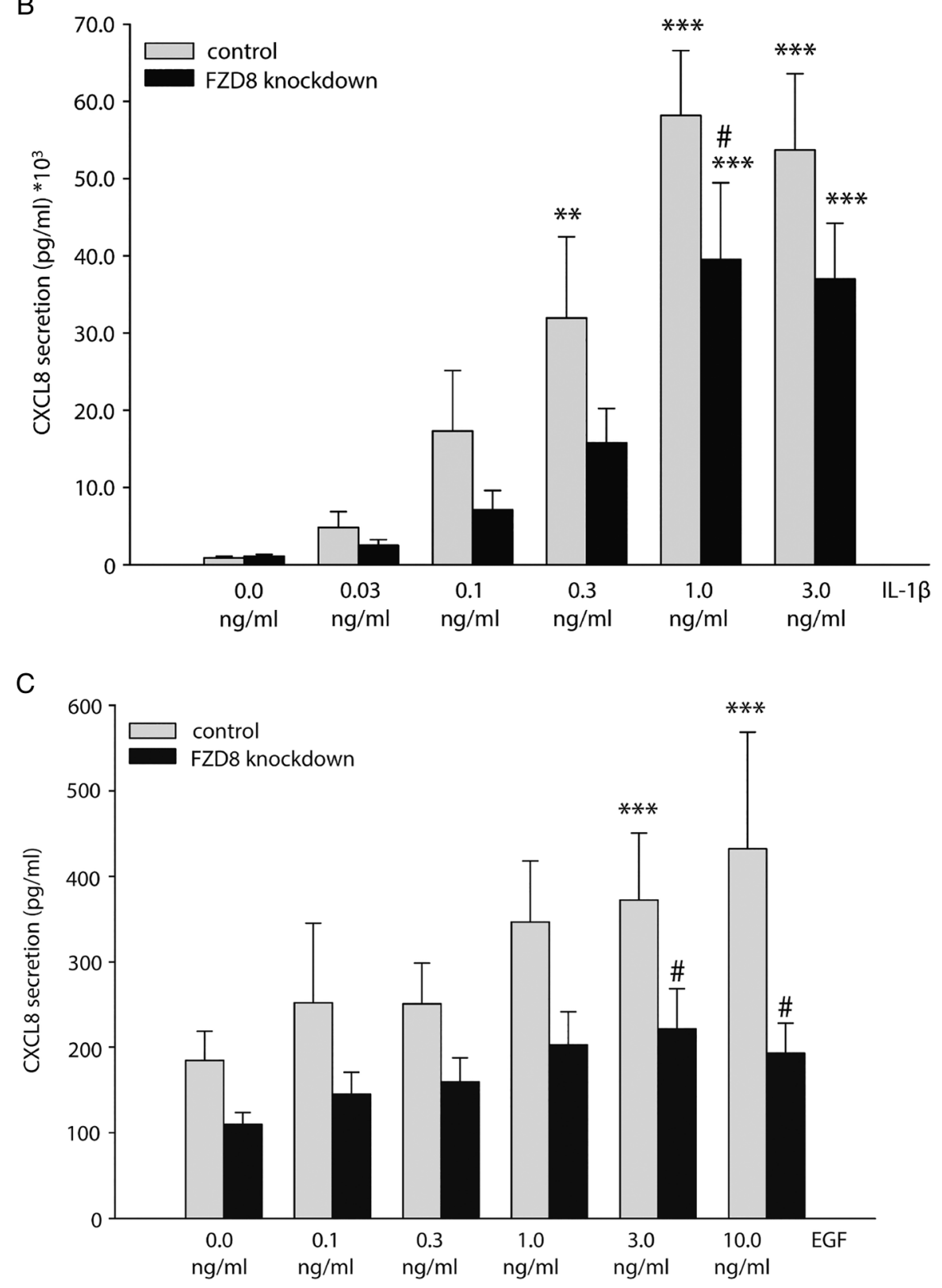
inducing mucus production. There are no biologicals used in COPD that target IL-6. Blocking CXCL8 by using a blocking antibody only or by blocking the CXCR2 receptor has been reported not to have a clinical impact. ${ }^{36}$ Our data show that targeting FZD8 on fibroblasts could prevent both the secretion of IL-6 and CXCL-8, leading to a reduction in inflammatory processes and mucus secretion. Therefore, FZD8 may provide a rational drug target for chronic bronchitis and the development of FZD8 receptor antagonists may be warranted.

In conclusion, our results indicate that FZD8 plays an important pro-inflammatory role in chronic bronchitis. Interestingly, the fibroblast appears to play a major role herein. We provide genetic and functional data in vitro and in vivo supporting the role of FZD8 in pro-inflammatory cytokine production, inflammatory cell recruitment and mucus hypersecretion. These findings show a potential important role for WNT signalling via FZD8 in fibroblasts in chronic bronchitis. Therefore, targeting FZD8 is a strategy worth pursuing for the treatment of chronic bronchitis.

\section{Author affiliations}

${ }^{1}$ Department of Molecular Pharmacology, University of Groningen, Groningen, The Netherlands

${ }^{2}$ Groningen Research Institute for Asthma and COPD (GRIAC), University of Groningen, University Medical Center Groningen, Groningen, The Netherlands

${ }^{3}$ Department of Pulmonology, University of Groningen, University Medical Center Groningen, Groningen, The Netherlands

${ }^{4}$ Department of Epidemiology, University of Groningen, University Medical Center Groningen, Groningen, The Netherlands

${ }^{5}$ Departments of Genetics and Pharmacogenomics, Merck Research Laboratories, Boston, Massachusetts, USA

${ }^{6}$ Center for Heart Lung Innovation, The University of British Columbia, Vancouver, British Columbia, Canada

${ }^{7}$ Respiratory Division, University of British Columbia, Vancouver, British Columbia, Canada

${ }^{8}$ Institut universitaire de cardiologie et de pneumologie de Québec, Québec City, Canada

${ }^{9}$ Department of Molecular Medicine, Laval University, Québec City, Canada

${ }^{10}$ Department of Pathology \& Medical Biology, University of Groningen, University Medical Center Groningen, Groningen, The Netherlands

Acknowledgements The authors would like to thank L.L.G. Šebek and I.S.T. Bos at the University of Groningen and the research staff at the Respiratory Health Network Tissue Bank of the FRQS for their valuable assistance.

Contributors Conception and design: AIRS, DSP, HM, IHH and RG. Laboratory work: AIRS (in vitro and in vivo studies); MHM (in vitro studies); C-AB and WT (primary fibroblast characterisation of patients with COPD). Data analysis and interpretation: AIRS, MHM, C-AB, DCN, DDS, WT, DSP, HM, IHH, RG, AED and HMB (genetic association study); MvdB and YB (eQTL analysis). Drafting of manuscript: AIRS, AED, MvdB, IHH and RG. Critically revising manuscript: MvdB, HMB, DCN, DDS, YB, C-AB, WT, DSP, HM, IHH and RG. All authors have read, reviewed and approved the final manuscript as submitted to take public responsibility for it.

Funding This work was supported by the Dutch Lung Foundation (3.2.10.042).

Competing interests The University of Groningen has received money for DSP regarding an unrestricted educational grant for research from Chiesi. Travel to ERS and/or ATS has been partially funded by Chiesi, GSK, and Takeda. Fees for consultancies were given to the University of Groningen by Boehringer Ingelheim, Chiesi, GSK, Takeda and TEVA. Travel and lectures in China paid by Chiesi. WT reports personal fees (all to the institution: UMCG) from Pfizer, GSK, Chiesi, and Roche Diagnostics/Ventana, and grants from Dutch Asthma Fund, all outside the submitted work. DCN is a full-time employee of Merck and Company.

Ethics approval The study protocol followed national ethical and professional guidelines ('Code of conduct; Dutch federation of biomedical scientific societies'; http://www.federa.org) for all lung tissue and explant cell culture studies in Groningen. For the genetics study on $\mathrm{CMH}$, approval by the local medical ethics committee and written informed consent from all patients were obtained (see online supplementary file).

Provenance and peer review Not commissioned; externally peer reviewed.

\section{REFERENCES}

1 Global Initiative for Chronic Obstructive Lung Disease (GOLD). 2014. http://www. goldcopd.org
2 Barnes PJ. Cellular and molecular mechanisms of chronic obstructive pulmonary disease. Clin Chest Med 2014:35:71-86.

$3 \mathrm{Kim}$ V, Criner GJ. Chronic bronchitis and chronic obstructive pulmonary disease. Am J Respir Crit Care Med 2013:187:228-37.

4 Vestbo J, Prescott E, Lange P. Association of chronic mucus hypersecretion with FEV1 decline and chronic obstructive pulmonary disease morbidity. Copenhagen City Heart Study Group. Am J Respir Crit Care Med 1996;153:1530-5.

5 Ramos FL, Krahnke JS, Kim V. Clinical issues of mucus accumulation in COPD. Int J Chron Obstruct Pulmon Dis 2014;9:139-50.

6 de Marco R, Accordini S, Cerveri I, et al. Incidence of chronic obstructive pulmonary disease in a cohort of young adults according to the presence of chronic cough and phlegm. Am J Respir Crit Care Med 2007;175:32-9.

7 Pongracz JE, Stockley RA. Wnt signalling in lung development and diseases. Respir Res 2006; 7:15

8 Baarsma HA, Spanjer Al, Haitsma G, et al. Activation of WNT/beta-catenin signaling in pulmonary fibroblasts by TGF-beta(1) is increased in chronic obstructive pulmonary disease. PLOS ONE 2011;6:e25450.

9 Kumawat $\mathrm{K}$, Menzen MH, Bos IS, et al. Noncanonical WNT-5A signaling regulates TGF-beta-induced extracellular matrix production by airway smooth muscle cells. FASEB J 2013;27:1631-43.

10 Aumiller V, Balsara N, Wilhelm J, et al. WNT/beta-catenin signaling induces IL-1 beta expression by alveolar epithelial cells in pulmonary fibrosis. Am J Respir Cell Mol Biol 2013;49:96-104

11 Baarsma HA, Meurs H, Halayko AJ, et al. Glycogen synthase kinase-3 regulates cigarette smoke extract- and IL-1 beta-induced cytokine secretion by airway smooth muscle. Am J Physiol Lung Cell Mol Physiol 2011;300:L910-19.

12 Baarsma HA, Königshoff M, Gosens R. The WNT signaling pathway from ligand secretion to gene transcription: molecular mechanisms and pharmacological targets. Pharmacol Ther 2013;138:66-83.

13 Heijink $I H$, de Bruin $H G$, van den Berge $M$, et al. Role of aberrant WNT signalling in the airway epithelial response to cigarette smoke in chronic obstructive pulmonary disease. Thorax 2013:68:709-16.

14 Jung YS, Lee HY, Kim SD, et al. Wnt5a stimulates chemotactic migration and chemokine production in human neutrophils. Exp Mol Med 2013;45:e27.

15 van lersel $\mathrm{CA}$, de Koning $\mathrm{HJ}$, Draisma G, et al. Risk-based selection from the general population in a screening trial: selection criteria, recruitment and power for the Dutch-Belgian randomised lung cancer multi-slice CT screening trial (NELSON). Int J Cancer 2007;120:868-74.

16 Dijkstra AE, Smolonska J, van den Berge M, et al. Susceptibility to chronic mucus hypersecretion, a genome wide association study. PLOS ONE 2014;9: e91621.

17 Hao K, Bossé Y, Nickle DC, et al. Lung eQTLs to help reveal the molecular underpinnings of asthma. PLoS Genet 2012;8:e1003029.

18 Lamontagne M, Timens W, Hao K, et al. Genetic regulation of gene expression in the lung identifies CST3 and CD22 as potential causal genes for airflow obstruction. Thorax 2014;69:997-1004

19 Brandsma CA, van den Berge M, Postma DS, et al. A large lung gene expression study identifying fibulin-5 as a novel player in tissue repair in COPD. Thorax 2015;70:21-32.

20 Brandsma CA, Timens W, Jonker MR, et al. Differential effects of fluticasone on extracellular matrix production by airway and parenchymal fibroblasts in severe COPD. Am J Physiol Lung Cell Mol Physiol 2013;305:L582-9.

21 Jacobs JP, Jones CM, Baille JP. Characteristics of a human diploid cell designated MRC-5. Nature 1970;227:168-70.

22 Kistemaker LE, Bos IS, Hylkema MN, et al. Muscarinic receptor subtype-specific effects on cigarette smoke-induced inflammation in mice. Eur Respir $J$ 2013:42:1677-88.

23 Brandsma CA, Hylkema MN, Luinge MA, et al. Nitrogen dioxide exposure attenuates cigarette smoke-induced cytokine production in mice. Inhal Toxicol 2008;20:183-9.

24 Caramori G, Adcock IM, Di Stefano A, et al. Cytokine inhibition in the treatment of COPD. Int J Chron Obstruct Pulmon Dis 2014;9:397-412.

25 Bautista MV, Chen Y, Ivanova VS, et al. IL-8 regulates mucin gene expression at the posttranscriptional level in lung epithelial cells. J Immunol 2009;183:2159-66.

26 Vestbo J. COPD: definition and phenotypes. Clin Chest Med 2014;35:1-6.

27 Knight D. Epithelium-fibroblast interactions in response to airway inflammation. Immunol Cell Biol 2001;79:160-4.

28 Fuchigami T, Kibe T, Koyama H, et al. Regulation of IL-6 and IL-8 production by reciprocal cell-to-cell interactions between tumor cells and stromal fibroblasts through IL-1alpha in ameloblastoma. Biochem Biophys Res Commun 2014:451:491-6.

29 Osei ET, Brandsma CA, Noordhoek JA, et al. Crosstalk between epithelium and fibroblasts; implications for COPD. Eur Respir J 2014;44(suppl 58):3899.

30 Spanjer AIR, Jansen SR, Baarsma HA, et al. TGF- $\beta_{1}$-induced extracellular matrix protein expression in human lung fibroblasts requires autocrine WNT signaling via the Frizzled-8 receptor. Am J Respir Crit Care Med 2012;185:5525 
31 Dann $C E$, Hsieh JC, Rattner $A$, et al. Insights into Wnt binding and signalling from the structures of two Frizzled cysteine-rich domains. Nature 2001;412:86-90.

$32 \mathrm{Nam}$ JS, Turcotte TJ, Smith PF, et al. Mouse cristin/R-spondin family proteins are novel ligands for the Frizzled 8 and LRP6 receptors and activate beta-catenin-dependent gene expression. J Biol Chem 2006;281:13247-57.

33 Mercurio S, Latinkic B, Itasaki N, et al. Connective-tissue growth factor modulates WNT signalling and interacts with the WNT receptor complex. Development 2004;131:2137-47.
34 Zhu W, Shiojima I, Ito Y, et al. IGFBP-4 is an inhibitor of canonical Wnt signalling required for cardiogenesis. Nature 2008;454:345-9.

35 Van Dijk EM, Menzen MH, Spanjer AIR, et al. WNT-5A and B Induce IL-6 and IL-8 Release Via TAK1 Signaling in Human Lung Fibroblasts. Am I Respir Crit Care Med 2015;191:A3662.

36 Seiberling M, Kamtchoua T, Stryszak P, et al. Humoral immunity and delayed-type hypersensitivity in healthy subjects treated for 30 days with MK-7123, a selective CXCR2 antagonist. Int Immunopharmacol 2013;17:178-83. 